\title{
Four recombinant pluripotency transcriptional factors containing a protein transduction domain maintained the in vitro pluripotency of chicken embryonic stem cells
}

\author{
YU MiaoYing ${ }^{1,2}$, LIAN Song ${ }^{1,2}$, HAN HongBing ${ }^{2}$, YU Kun ${ }^{1,2}$, LI GuiGuan $^{2}$, \\ LIAN ZhengXing ${ }^{1,2 *}$ \& LI Ning $^{1 *}$ \\ ${ }^{1}$ National Key Laboratory of AgroBiotechnology, China Agricultural University, Beijing 100193, China; \\ ${ }^{2}$ Laboratory for Molecular and Cellular Genetics, Collage of Animal Science and Technology, China Agriculture University, Beijing 100193, \\ China
}

Received May 9, 2012; accepted October 31, 2012

\begin{abstract}
Long-term in vitro maintenance of embryonic stem cell (ESC) pluripotency enables the pluripotency and differentiation of ESCs in animals to be investigated. The ability to successfully maintain and differentiate chicken embryonic stem cells (cESCs) would provide a useful tool for avian biology research and would be a resource directly applicable to agricultural production. In this study, endogenous chicken pluripotency transcription factors, POUV, Sox-2, Nanog and Lin28 were cloned and expressed as recombinant proteins containing a nine consecutive arginine protein transduction domain (PTD). cESCs were cultured with these recombinant proteins to maintain cESC pluripotency in vitro. Cultured cESCs exhibited typical characteristics of pluripotency, even after six generations of rapid doubling, including positive staining for stage-specific embryonic antigen I, and strong staining for alkaline phosphatase. Expression levels of the pluripotency markers, POUV, Nanog, C-Myc, Sox-2 and Lin28 were the same as in uncultured stage $\mathrm{X}$ blastoderm cells, and most significantly, the formation of embryoid bodies (EBs) by 6th generation cESCs confirmed the ability of these cultured cESCs to differentiate into cells of all three embryonic germ layers. Thus, transcription factors could be translocated through the cell membrane into the intracellular space of cESCs by using a PTD of nine consecutive arginines and the pluripotency of cESCs could be maintained in vitro for at least six generations.
\end{abstract}

recombinant pluripotency factors, protein transduction domain, chicken ESC, pluripotency

Citation: Yu M Y, Lian S, Han H B, et al. Four recombinant pluripotency transcriptional factors containing a protein transduction domain maintained the in vitro pluripotency of chicken embryonic stem cells. Sci China Life Sci, 2013, 56: 40-50, doi: 10.1007/s11427-012-4426-4

Embryonic stem cells (ESCs) have been intensively researched since they were first derived from the inner cell mass (ICM) of mouse blastocysts in 1981 [1,2], and ESCs from many species have now been derived. Chickens are an important agricultural poultry species and also a vital animal research model for many biological processes. Chicken ESCs (cESCs) have been demonstrated to have pivotal util-

*Corresponding author (email: lianzhx@cau.edu.cn; ninglcau@cau.edu.cn) ity in transgenic poultry production, bird species conservation, and medical protein production [3]. The X stage blastoderm of chickens is the source of cESCs [3]. Cells separated from the $\mathrm{X}$ stage blastoderm of chickens exhibit typical ESC characteristics, including: positive staining for alkaline phosphatase (AKP) and stage-specific embryonic antigen I (SSEA-I), expression of pluripotency factors, such as POUV, Nanog, C-Myc, Sox-2 and Lin28, and differentiation potential toward all three embryonic germ layers via 
EB formation in vitro or chimera animal production in vivo [3-5]. Previous studies of cESCs have developed their potential, including the introduction of feeder cells, the employment of mouse and human cytokines and growth factors, and the cloning and functional studies of chicken cytokines, such as leukocyte inhibitory factor (LIF), and pluripotency genes, such as POUV and Nanog [3,4,6]. Although some culture methods have reported several generations of cESCs in vitro, these results could not be reliably reproduced, indicating unstable experimental protocols [6-8]. Development of a practical, stable, and reproducible long-term cultured cESC line is still a big challenge. Unlike for mice and humans, an in vitro chicken ESC culture line has not yet been reported [2,9], indicating the differences of ESC characteristics among species.

The pluripotency state of ESCs has been intensively studied and there is a significant, although incomplete, understanding of pluripotency at the molecular level. Research on induced pluripotency stem cells (iPSCs) has provided a new avenue and methodology to detect and understand the molecular mechanism of pluripotency in ESCs, in addition to highlighting the functions of pluripotency factors [10]. While some problems have been addressed using this technique, the safety of iPSC generation has been a significant concern because of the viral-mediated method used to generate iPSCs; the risk of reactivation from pseudotype virus back to wide-type virus has not been totally eliminated $[11,12]$. The inconvenient and troublesome procedures associated with this DNA insertion method have been another limitation because results are difficult to replicate and can be unstable. Thus, many studies have been performed to develop new methods [13-15].

The protein transduction domain (PTD) is a short peptide composed of part of the HIV-1 Tat sequence or a peptide of consecutive hydrophobic amino acids. Previous medical and pharmacological studies have demonstrated the high efficiency of PTDs as a means to quickly deliver macromolecules through cell membranes into the intracellular space of target cells [16-19].

Reports from two research groups provided a method for iPSC generation free of DNA insertion based on recombinant pluripotency proteins fused with a PTD consisting of 9 or 11 consecutive arginines [20,21]. This made iPSC generation safe and convenient: it established a stable tool for ESC research, and suggested the possibility of using pluripotency factors in ESC cultures. Although there has not been an iPSC line generated for chicken, the attractive and powerful functions of pluripotency factors should be investigated in ESCs of different species. These pluripotency factors can re-establish pluripotency from the differentiated state; however, it is not known whether they prevent pluripotency from being lost. Here, we aim to determine if pluripotency factors in the form of recombinant proteins with a PTD can maintain the pluripotency of cESCs cultured in vitro.

\section{Materials and methods}

\subsection{Plasmid construction}

\subsection{1 pEGFP9R}

Primers to PCR amplify EGFP coding sequence (CDS) were designed. Nine consecutive arginines at the $5^{\prime}$ end of the antisense primer and restriction endonuclease sites at the $5^{\prime}$ end of both sense and antisense primers were incorporated. pEGFP-NI was used as a template. PCR products were subcloned into pEGFP-NI, from which the EGFP sequence has been removed, resulting in the plasmid pEGFP9R.

\subsection{2 pPF9R-IRES}

Primers to PCR amplify CDSs of pluripotency proteins, POUV-9R, Sox-2-9R, Nanog-9R and Lin28-9R were designed incorporating nine consecutive arginines at the $5^{\prime}$ end of the antisense primers and restriction endonuclease sites at the $5^{\prime}$ end of both sense and antisense primers. POUV, Sox-2, Nanog and Lin28 CDS were amplified by RT-PCR from cESCs total RNA using these primers. PCR products were subcloned into pEGFP-IRES2, resulting in pPF9RIRES plasmids, including pPOUV9R-IRES, pSox-2-9RIRES, pNanog9R-IRES and pLin28-9R-IRES.

1.2 Evaluation of the efficiency of the nine consecutive arginine PTD in translocating exogenous recombinant protein into the intracellular space

pEGFP9R and pEGFP-NI (as control) were transfected into 293FT cells using the calcium phosphate transfection method. The two transfected cell cultures were then ultrasonically disrupted, and the extracts were filtered and concentrated through Amicon Ultra-15 (Millipore, Billerica, MA, USA). The cell lysate concentrate containing PTDfused recombinant EGFP protein or non-PTD-fused EGFP protein was then added to cESC culture medium. Six hours later, EGFP signal was assessed under a fluorescence microscope to evaluate the efficiency of the PTD in translocating exogenous recombinant protein into the intracellular space.

\subsection{Recombinant pluripotency protein production}

pPF9R-IRES plasmids were transfected into 293FT cells using the calcium phosphate transfection method. Approximately $80 \mu \mathrm{g}$ of each pPF9R-IRES plasmid was used to transfect $4 \times 10^{8} 293 \mathrm{FT}$ cells. After three days, when a green fluorescence signal from the transfected 293FT cells was observed under a fluorescence microscope, cells were washed twice with PBS and then ultrasonically disrupted. Cell extracts were filtered and concentrated through Amicon Ultra-15 (Millipore) and subsequently stored at $-80^{\circ} \mathrm{C}$. The presence of recombinant pluripotency proteins 
in these cell extracts was evaluated by western blot analysis.

\subsection{Western blot analysis}

Concentrated cell extracts containing different recombinant pluripotency proteins were subjected to $12 \%$ SDSpolyacrylamide gel electrophoresis (PAGE) and transferred to PVDF membranes (Millipore). After blocking with a solution of dried skimmed milk, membranes were incubated with 1:1000 dilutions of the following primary antibodies: rabbit anti-human POUV antibody (R\&D, Minneapolis, MN, USA), mouse anti-human Nanog antibody (R\&D), rabbit anti-human Sox-2 antibody (R\&D), and mouse anti-human Lin28 antibody (R\&D). The membranes were then incubated with a 1:5000 dilution of the appropriate horseradish peroxidase-conjugated secondary antibody: goat anti-rabbit immunoglobulin G (IgG) (Kangchen, Beijing, China) and goat anti-rabbit immunoglobulin $\mathrm{G}$ (IgG) (Kangchen). Detection was proformed using an enhanced-chemiluminescent substrate (Kangchen).

\subsection{Cell culture and addition of recombinant pluripo- tency proteins}

Dulbecco's modified Eagle's medium (DMEM, Invitrogen, California, CA, USA), supplemented with $2 \mathrm{mmol} \mathrm{L}^{-1}$ L-glutamine (Invitrogen), $1 \mathrm{mmol} \mathrm{L}^{-1} \beta$-mercaptoethanol, $1 \times$ non-essential amino acids (NEAA, Invitrogen), $10 \%$ fetal bovine serum (FBS, Hyclone, Thermo Scientific, Logan, UT, USA), $100 \mathrm{U} \mathrm{mL}^{-1}$ penicillin, and $100 \mu \mathrm{g} \mathrm{mL}^{-1}$ streptomycin (Invitrogen) was used as a basic culture medium. cESCs were cultured in a 96-well culture dish using the basic culture medium, supplemented with each recombinant pluripotency protein at $1.2 \mu \mathrm{g} \mathrm{mL}^{-1}$. Mouse LIF (R\&D) and human bFGF (Invitrogen) were added at concentrations of $1500 \mathrm{U} \mathrm{mL}^{-1}$ and $1 \mathrm{ng} \mathrm{mL}^{-1}$, respectively.

\subsection{Cell proliferation analysis}

To assess the proliferation of cells, we used Cell Counting Kit-8 (CCK-8, Dojindo Laboratories, Tokyo, Japan) and propidium iodide (PI) staining. The former tests for cell viability, as determined by the CCK- 8 OD value, while the later tests for cell viability and cell apoptosis.

\subsection{Alkaline phosphatase reaction}

Cells were fixed with cold $4 \%$ paraformaldehyde for $15 \mathrm{~min}$ after washing with PBS several times. The AKP reaction was performed at $37^{\circ} \mathrm{C}$ for $30 \mathrm{~min}$ by adding $10 \mu \mathrm{L} \mathrm{AKP}$ staining solution $\left(100 \mathrm{mmol} \mathrm{L}^{-1} \mathrm{NaCl}, 100 \mathrm{mmol} \mathrm{L}^{-1}\right.$ Tris-HCl (pH 9.5), $5 \mathrm{mmol} \mathrm{L}^{-1} \mathrm{MgCl}_{2}, 1 \mathrm{mg} \mathrm{mL} \mathrm{mBT}^{-1} \mathrm{NB}$, and $0.1 \mathrm{mg} \mathrm{mL} \mathrm{mCIP}^{-1}$ to each well. The reaction was stopped by washing cells with PBS.

\subsection{Immunofluorescence analysis of SSEA-I}

After fixing with cold $4 \%$ paraformaldehyde for $15 \mathrm{~min}$ at room temperature, cells were blocked in $1 \%$ BSA, incubated with fluorescent isothiocyanate-conjugated human anti-SSEA-I monoclonal antibody (R\&D) diluted 1:500 in blocking agent for $1 \mathrm{~h}$ at room temperature, and then washed twice with PBS. Stained cells were observed under an inverted fluorescence microscope.

\subsection{Real-time RT-PCR analysis of pluripotency marker genes}

To analyze mRNA levels, cells were treated with TRIzol reagent (Invitrogen) and extracted total RNA was reverse transcribed. Real-time PCR analyses were then performed in triplicate using SYBR Green I and a DNA Engine Opticon (MJ Research, Waltham, Massachusetts, USA).

\subsection{Embryoid body formation by cultured cESCs}

After six generations, cultured cESCs were dissociated and cultured in suspension without any anti-differentiation factors on smooth surface dishes coated with $1 \%$ agarose gel to prevent cell adhesion to the plastic surfaces.

\subsection{Statistical analysis}

Results are reported as mean \pm standard error (SE). We tested the data for statistical significance using Student's $t$-test. A $P$-value less than 0.05 was considered significant. All experiments were repeated at least three times to ensure reproducibility.

\section{Results}

\subsection{The PTD fusion protein was efficiently translocat- ed into the intracellular space of cESCs}

To test the efficiency of the nine consecutive arginine PTD to deliver proteins into cESCs, a PTD efficiency test vector, pEGFP9R, was constructed (Figure 1A, Table 1). 293FT cells were transfected with pEGFP9R, cultured and then ultrasonically disrupted. Isolated cell extracts, containing recombinant EGFP fused with the PTD, were added to cESCs culture media. 293FT cells were also transfected with pEGFP-NI and the expressed no-PTD-fusion EGFP protein was added in cESCs culture medium as a control. Based on fluorescence microscopy observations, approximately $70 \%-80 \%$ of cESCs contained translocated recombinant EGFP, while translocation of the no-PTD-fused EGFP was not detected (Figure 1B). This indicated that the nine consecutive arginine PTD functioned with high efficiency in delivering foreign proteins into the cytoplasm of cESCs. Thus, recombinant pluripotency proteins with a nine 


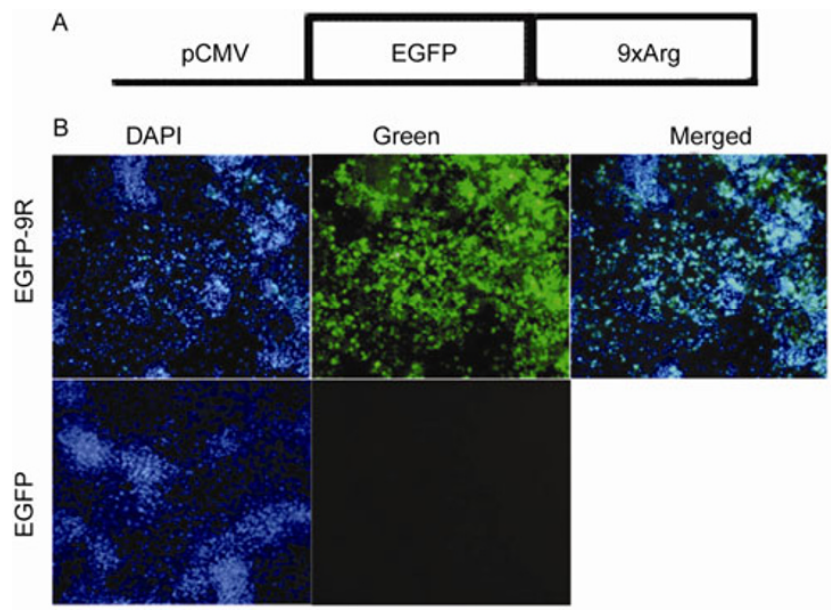

Figure 1 Nine consecutive arginine PTD-fused-EGFP efficiently translocated into the intracellular space of cESCs. A, Schematic representation of the PTD efficiency test vector (pEGFP9R). EGFP was fused with a nine consecutive arginine sequence. A CMV promoter was used to drive the EGFP-PTD cassette. B, 293FT cell extract containing PTD-fused-EGFP (EGFP-9R) or 293FT cell extract containing no-PTD-fused EGFP (EGFP) was added to cESC culture media. Green fluorescence was observed under a fluorescence microscope $6 \mathrm{~h}$ later. Images are at $100 \times$ magnification.

consecutive arginine PTD should be able to enter the cytoplasm of cultured cESCs.

\subsection{Production of recombinant pluripotency proteins and addition to cESC culture media}

Recombinant pluripotency protein expression vectors, pPF9R-IRES, were constructed (Figure 2A, Table 1), such that the pluripotency proteins were expressed synchronously with EGFP in transfected 293FT cells. By EGFP signal observation and Western blot analysis, the four recombinant pluripotency proteins were demonstrated to be expressed in 293FT cells and to be contained in concentrated 293FT cell extracts (Figure 2B).

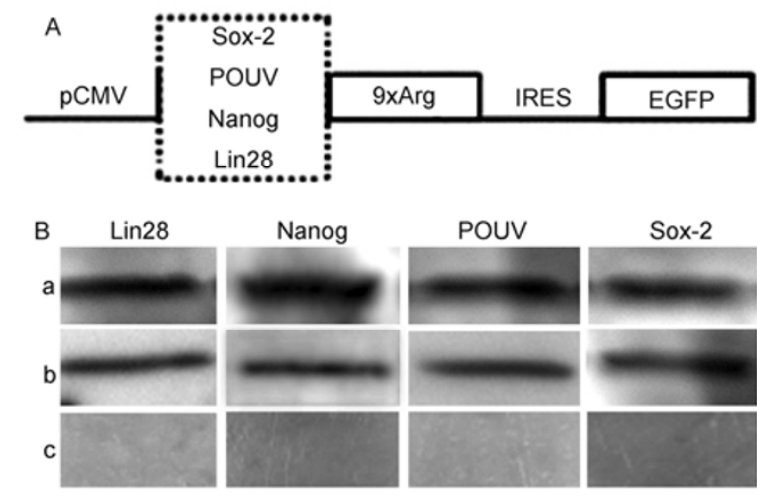

Figure 2 PTD fused recombinant pluripotency factor expression vectors (pPF9R-IRES) and expression of recombinant pluripotency proteins. A, Schematic representation of pluripotency protein expression vector (pPF9R-IRES): the four pluripotency factors (Sox-2, POUV, Nanog and Lin28) were fused with a sequence of nine consecutive arginines. PF-PTD and EGFP coding sequences were separated by an internal ribosomal entry site (IRES) and expression was driven by a CMV promoter. B, Correct processing of each pluripotency protein was confirmed by Western blot analysis. a, extracts from 293FT cells transfected with the four different pPF9-IRES plasmids were reacted with antibodies against the four pluripotency factors; $b$, non-cultured X blastoderm cells were reacted with antibodies against the four pluripotency factors; c, extracts from 293FT cells transfected with pEGFP-IRES2 were reacted with antibodies against the four pluripotency factors.

\subsection{Four recombinant pluripotency proteins inhibited differentiation of cESCs in vitro for three generations}

CCK-8 and PI tests showed that the four recombinant pluripotency proteins inhibited the differentiation of cESCs. Cells cultured with the four pluripotency proteins sustained high proliferation rates in vitro for up to three generations (Figure 3A); however, after three generations, cells underwent apoptosis or died, and no differentiated morphological shapes were observed (Figure 3B). Expression of pluripotency marker genes was detected in cESCs before the third passage (Figure 3C), while expression of differentiation

Table 1 Primers used to amplify EGFP and pluripotency protein coding sequences ${ }^{\text {a) }}$

\begin{tabular}{|c|c|}
\hline Primer name & Primer sequence \\
\hline EGFP9R sense & 5'-CCGGGATCCGCCATGGTGAGCA AGGGCGAGGA-3' \\
\hline EGFP9R antisense & $\begin{array}{l}\text { 5'-TTTGCGGCCGCTTAGCGGCGTCTGCGTCTGCGGCGTCTGCG } \\
\text { CTTGTACAGCTCGTCCATG-3' }\end{array}$ \\
\hline POUV9R sense & 5'-CCGGAATTCCGCCACCATGCATGTAAAAGCCAAAAACC-3' \\
\hline POUV9R antisense & $\begin{array}{l}\text { 5'-CGGGG_tA_CCTCAGCGGCGTCTGCGTCTGCGGCGTCTGCGGT } \\
\text { GGCTGCTGTTGTTCATGG-3' }\end{array}$ \\
\hline Nanog9R sense & 5'-CCGGAATTCCGCCACCATGAGCGCTCACCTGGCCATG-3' \\
\hline Sox-2 9R sense & 5'-CCGGAATTCCGCCACCATGATGGAAACCGAGCTGAAACC-3' \\
\hline Sox-2 9R antisense & $\begin{array}{l}\text { 5'-CGGGG_ATCC TTAGCGGCGTCTGCGTCTGCGGCGTCTGCGC } \\
\text { ATATGTGATAGAGGGAGTG-3' }\end{array}$ \\
\hline Lin28 9R sense & 5'-CGGG̣GTACC_GCCACCATGGGGTCTGTTTCCAACCAG-3' \\
\hline Lin28 9R antisense & $\begin{array}{l}\text { 5'-CGGGGA_t_C_TCAGCGGCGTCTGCGTCTGCGGCGTCTGCGT } \\
\text { TCCCGGGTTTCGGGGAGC-3' }\end{array}$ \\
\hline
\end{tabular}

a) Dashed underscore indicates sequence of endonuclease sites. Solid underscore indicates sequence of nine consecutive arginines. 

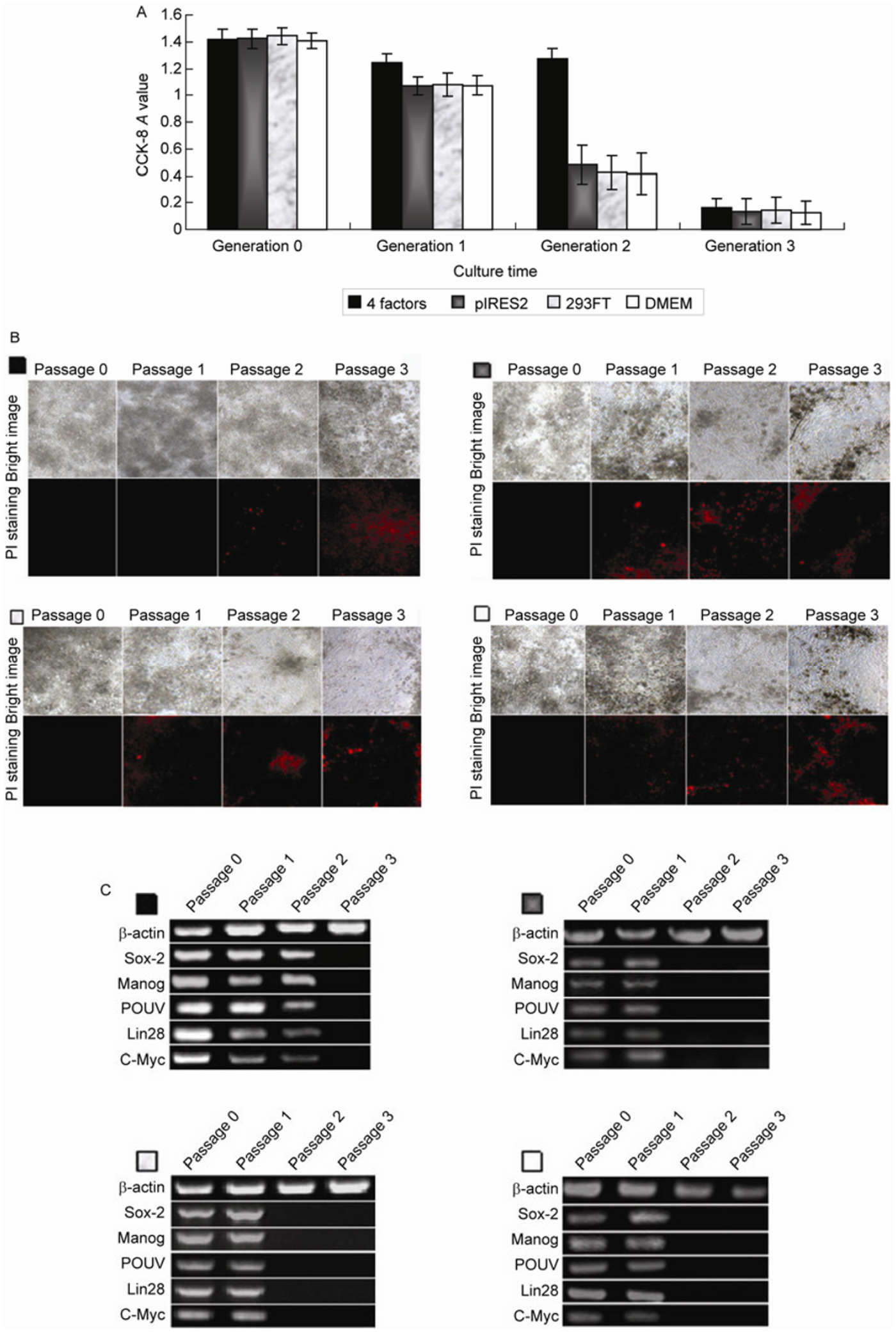

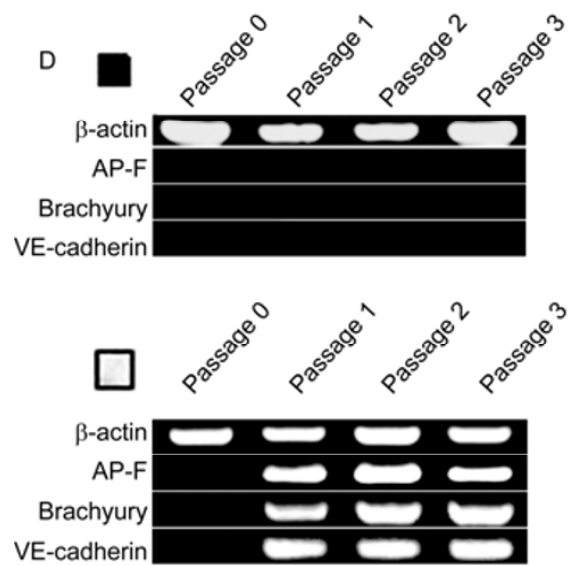
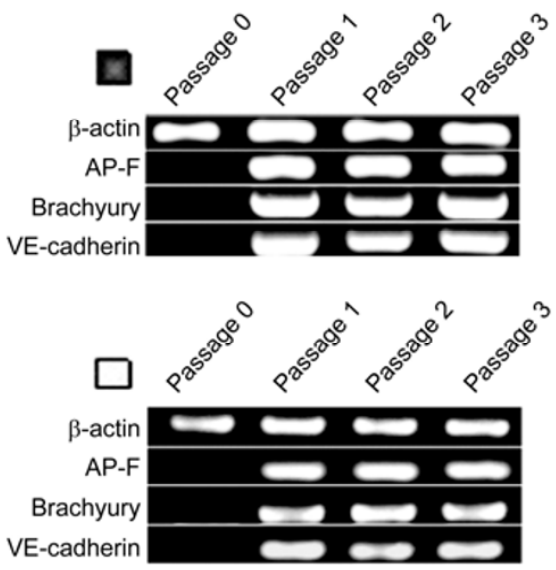

Figure 3 Four recombination pluripotency proteins inhibit differentiation of cESCs in vitro for three generations. A, Viability of in vitro cultured cESCs was tested using CCK-8. $\mathbf{\square}$, Medium containing the four pluripotency proteins (basic culture medium was supplemented with cell extracts from 293FT cells transfected with pPF9R-IRES plasmids); $\square$, control medium 1 (basic culture medium supplemented with cell extracts from $293 \mathrm{FT}$ cells transfected with pIRES2-EGFP); $\square$, control medium 2 (basic culture medium supplemented with cell extracts from 293FT cells without any plasmid transfection); $\square$, control medium 3 (basic culture medium: DMEM supplemented with 10\% FBS). B, PI staining and morphological characteristics of cESCs cultured in vitro.

$\mathbf{\square}$, cESCs cultured in medium containing the four pluripotency proteins; $\boldsymbol{\square}$, cESCs cultured in control medium 1 ; $\square$, cESCs cultured in control medium 2 ; $\square$, cESCs cultured in control medium 3. Images are 100× magnification. C, Expression of pluripotency marker genes in cESCs cultured in vitro. $\mathbf{\square}$, cESCs passaged 0-3 times cultured in medium containing the four pluripotency proteins; $\square$, cESCs passaged 0-3 times cultured in control medium 1; $\square$, cESCs passaged 0-3 times cultured in control medium 2; $\square$, cESCs passaged 0-3 times cultured in control medium 3. D, Expression of differentiation marker genes in cESCs cultured in vitro. $\mathbf{\square}$, cESCs passaged 0-3 times cultured in medium containing the four pluripotency proteins; $\mathbf{\square}$, cESCs passaged 0-3 times cultured in control medium $1 ; \square$, cESCs passaged $0-3$ times cultured in control medium 2 ; $\square$, cESCs passaged 0-3 times cultured in control medium 3 .

marker genes was not detected (Figure 3D). These results showed that there was no tendency towards differentiation in cESCs cultured with the four recombinant pluripotency proteins. Control cultures were grown in three different media: basic medium containing pEGFP-IRES2 transfected 293FT cell extracts, basic medium containing no plasmidtransfected 293FT cell extracts, and basic medium only. In these control media, proliferation rates of cultured cESCs were reduced after just one generation (Figure 3A) and differentiated morphological shapes were observed after just one passage (Figure 3B). Moreover, in these cESCs, the expression of pluripotency marker genes was not detected after one passage (Figure 3C), while expression of differentiation marker genes was detected after one passage (Figure $3 \mathrm{D})$. All of these observations suggest that the four pluripotency proteins inhibited in vitro differentiation of cultured cESCs for three generations.

\subsection{Four recombinant pluripotency proteins combined} with LIF and bFGF promoted the proliferation of cESCs for seven generations and typical pluripotency characteristics were maintained

To prolong the culture time of cESCs, mouse cytokine, LIF, and human growth factor, bFGF, were added to the culture medium, because they have the ability to promote proliferation of mESCs and hESCs. CCK-8 results indicated that cESCs cultured with a combination of the four pluripotency factors, LIF and bFGF could proliferate in vitro for seven generations. Media containing the four pluripotency proteins and either LIF or bFGF could promote proliferation of cultured cESCs for five generations, while media containing only LIF and bFGF could promote proliferation for just three generations (Figure 4A). Moreover, the pluripotency assays, SSEA-I immunofluorescence staining and AKP reaction staining, showed that cESCs cultured with the four pluripotency proteins, LIF, and bFGF still expressed the ESC-specific surface antigen, SSEA-I, and endogenous AKP even after seven passages (Figure 4B). In comparison, cESCs cultured with LIF and bFGF only were negative for the two pluripotency markers after three passages and, together with the morphological images of these cells, these results indicated that cESCs cultured with only LIF and bFGF lost pluripotency characteristics and underwent differentiation after just one passage (Figure 4C). Quantitative RT-PCR (Table 2) showed that the mRNA levels of endogenous pluripotency genes, such as POUV, Nanog, Sox-2, Lin28 and C-Myc, were not diminished in cultured cESCs until the 7 th passage, when a rapid decline of Lin28 expression occurred. This decrease was followed by decreases in the expression of the other four genes and a stop in proliferation (Figure 4D). These results demonstrated that the four recombinant pluripotency proteins combined with LIF and bFGF could maintain pluripotency of cESCs in vitro and support their proliferation for seven generations. 

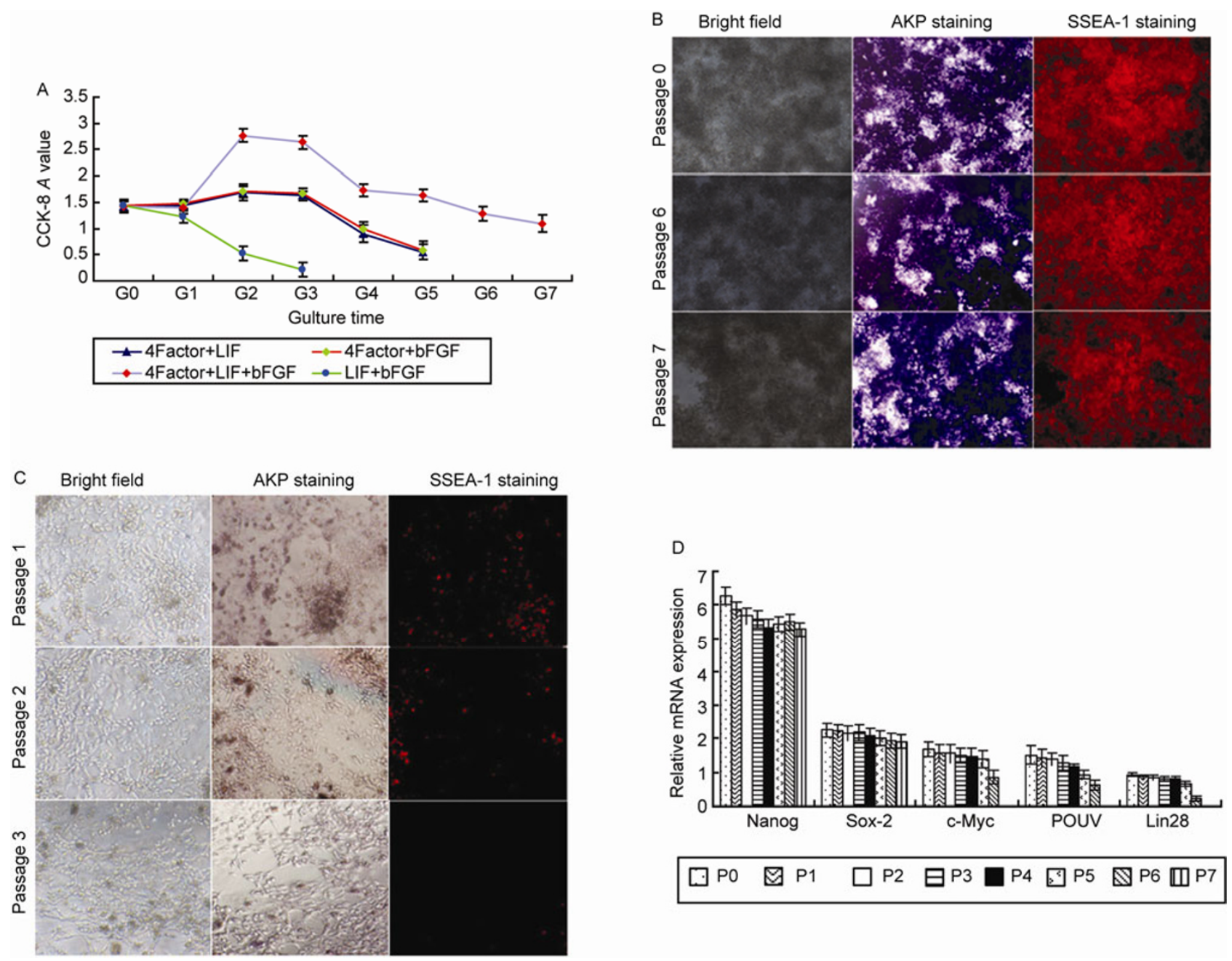

Figure 4 Four recombination pluripotency proteins combined with LIF and bFGF promoted the proliferation of cESCs for seven generations and typical pluripotency characteristics were maintained. A, Viability of cultured cESCs was tested using CCK-8. $\rightarrow$, Four pluripotency proteins and LIF; $-\downarrow$, four pluripotency proteins and bFGF; $\rightarrow-$, four pluripotency proteins, LIF and bFGF; $\rightarrow-$, LIF and bFGF only. B, cESCs cultured for 0, 6, and 7 passages with pluripotency proteins, LIF, and bFGF were used to assess morphology, AKP activity, and SSEA-I expression to determine pluripotency. Images are 100x magnification. C, cESCs cultured for 1, 2, and 3 passages with LIF and bFGF only. Morphology, AKP activity, and SSEA-I expression were assessed to determine pluripotency. Images are 100× magnification. D, mRNA levels of pluripotency genes in cESCs cultured for 7 passages with the four recombinant pluripotency proteins, LIF, and bFGF were assessed by quantitative RT-PCR.

Table 2 Primers used to quantitative RT-PCR amplify pluripotency marker genes

\begin{tabular}{|c|c|}
\hline Primer name & Primer sequence \\
\hline POUV sense & 5'-AAAGGATTGCGAAATGCCCG-3' \\
\hline POUV antisense & 5'-CTGAGTGAAGCCCAGCATGA-3' \\
\hline Nanog sense & 5'-ATCCGTTCATGGCTGTGGAG-3' \\
\hline Nanog antisense & 5'-CTGATGCCGTACAGGAGAGC-3' \\
\hline Sox-2 sense & 5'-CTGGACCAACGGAGGCTATG-3' \\
\hline Sox-2 antisense & 5'-AGACGAGGAGGTGACTACGG-3' \\
\hline Lin28 sense & 5'-AGGTTTGGAATCCATCCGGG-3' \\
\hline Lin28 antisense & 5'-AGCTGTGCATGTCCTCCTTC-3' \\
\hline C-Myc sense & 5'-TTCCATCGGTCAGGACACAG-3' \\
\hline C-Myc antisense & 5'-TCATTACGCCTCTGACGCTC-3' \\
\hline
\end{tabular}




\section{5 cESCs cultured for six passages still maintained differentiation potential and telomerase activity}

Maintaining the ability to differentiate into cells of the three embryonic germ layers is the most significant evidence possible to demonstrate ESC pluripotency. In our study, cESCs cultured for six generations exhibited the ability to differentiate into cells of the three embryonic germ layers through EB formation. cESCs cultured for six generations, and then put into a suspension culture environment for four days resulted in the generation of several EB structures (Figure 5A). Expression of differentiation genes by the formed EB structures was detected by RT-PCR (Table 3). Cells of all three embryonic germ layers are represented by expression of these genes (Figure 5B), including endoderm derivatives (APF, GATA6, GATA4), mesoderm derivatives (Brachyury, CD34, NKX2-5), and ectoderm derivatives (Vasa, VEcadherin, $\mathrm{Cdx}-2$ ). Immortality is another significant property of ESC pluripotency, which relies on telomerase activity to prevent telomere shortening. Telomerase reverse tran- scriptase (TERT) expression is often used as an estimate of telomerase activity. In our study, telomerase activity in cESCs cultured from zero to six passages was assessed by assaying TERT expression at the mRNA level using RT-PCR. The results demonstrated that telomerase activity in our cultured cESCs remained positive as passage number increased (Figure 5C). These results indicated that cultured cESCs were still pluripotent after six generations and that they exhibited the potential to be cultured for a longer times.

\section{Discussion}

Pluripotency factors have not previously been reported to be used in culturing ESCs. Here we consider the difficulties of using pluripotency factors as part of a culture medium. By observing recombinant EGFP protein, the sequence of nine consecutive arginines was demonstrated to be a highly effi-

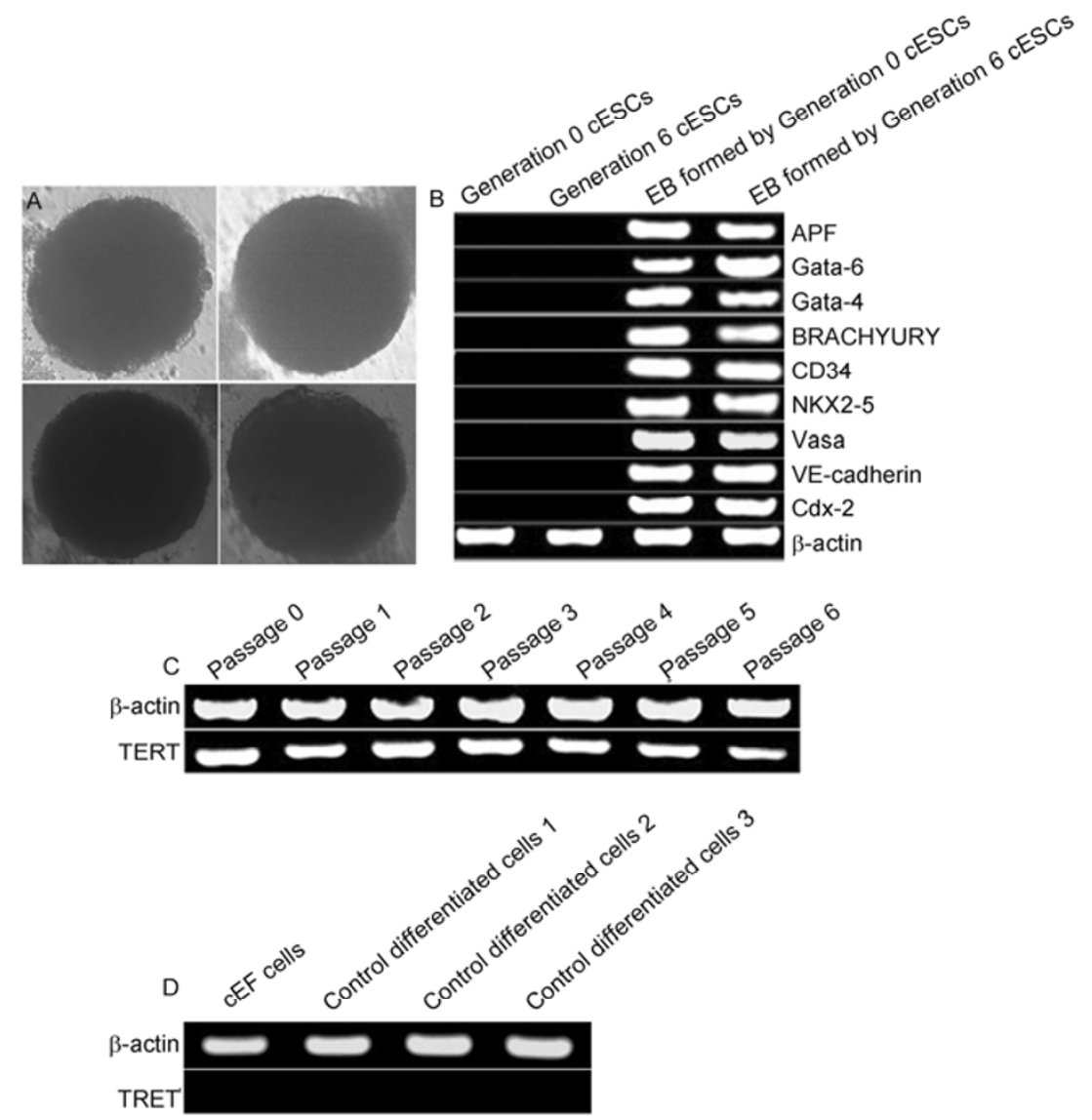

Figure 5 Ability of cESCs to differentiate into cells of the three embryonic layers in vitro. A, cESCs cultured in vitro formed three-dimensional, multicellular spheroid aggregates of EB structures by the 6th generation. Images are 100× magnification. B, Expression analysis of differentiation genes using total RNA extracted from EB formations and primers of genes expressed in the three embryonic germ layers, $\beta$-actin was employed as a control. C, TERT expression was detected by RT-PCR of cESCs passaged 0-6 times in the presence of the four recombinant pluripotency proteins to determine telomerase activity, $\beta$-actin was employed as a control. D, TERT expression was assayed by RT-PCR in chicken embryonic fibroblast cells (cEFCs) and in differentiated cells from cESCs cultured in control media in the absence of recombinant pluripotency proteins to determine telomerase activity, $\beta$-actin was employed as a control. 
Table 3 Primers used to amplify genes expressed in cells associated with the three embryonic germ layers, TRET, and $\beta$-actin

\begin{tabular}{|c|c|}
\hline Primer name & Primer sequence \\
\hline AFP sense (endoderm derivatives) & 5'-CCTGACATCGAGGTGATCCT-3' \\
\hline AFP antisense (endoderm derivatives) & 5'-CAAAGAGACCCAGCAGGAAG-3' \\
\hline Gata6 sense (endoderm derivatives) & 5'-CCGACCACTTGCTATGAAAAA-3' \\
\hline Gata6 antisense (endoderm derivatives) & 5'-CAGCCCATCTTGACCTGAATA-3' \\
\hline Gata4 sense (endoderm derivatives) & 5'-TGAGAAAAGAGGGCATTCAGA-3' \\
\hline Gata4 antisense (endoderm derivatives) & 5'-GCAGGATGAATTGAAGATCCA-3' \\
\hline NKX2-5 antisense (mesoderm derivatives) & 5'-CTGCTGCTTGAACCTTCTCT-3' \\
\hline BRACHYURY sense (mesoderm derivatives) & 5'-ACGCCATGTACTCCTTCCTG-3' \\
\hline BRACHYURY antisense (mesoderm derivatives) & 5'-TGTTGGTGAGCTTGACCTTG-3' \\
\hline CD34 sense (mesoderm derivatives) & 5'-ACCATGGGTTCCAGCACTAC-3' \\
\hline CD34 antisense (mesoderm derivatives) & 5'-TGCTTGTGTTCTCTGGATGC-3' \\
\hline Vasa sense (ectoderm derivatives) & 5'-TGTGGCAACTTCGGTAGCAT-3' \\
\hline Vasa antisense (ectoderm derivatives) & 5'-TGGACAGCCATTCTTCCACC-3' \\
\hline VE-Cadherin sense (ectoderm derivatives) & 5'-TCTTGGCTCCAGCATTCTCT-3' \\
\hline VE-Cadherin antisense (ectoderm derivatives) & 5'-CCATCGTACCCTTGCACTTT-3' \\
\hline Cdx2 sense (ectoderm derivatives) & 5'-TCAAAACCAGGACGAAGGAC-3' \\
\hline $\mathrm{Cd} 22$ antisense (ectoderm derivatives) & 5'-CCAGATTTTCACCTGCCTCT-3' \\
\hline TRET sense & 5'-GCCATCCCCAGGGTTTGTTA-3' \\
\hline TRET antisense & 5'-ACGTGCAGTAACAGTGCTGA-3' \\
\hline$\beta$-actin sense & 5'-TTACCCACACTGTGCCCATC-3' \\
\hline$\beta$-actin antisense & 5'-TGACCTGACCATCAGGGAGT \\
\hline
\end{tabular}

cient tool to translocate fusion proteins into the intracellular space of cESCs. This correlated with previous reports from medical and pharmacology studies showing that many medical proteins conjugated with PTDs, such as HIV-1 Tat or poly-hydrophobic amino acids, can be delivered into cells and remain functionally intact, with efficiencies of about $70 \%-90 \%$ [16-22]. The 70\%-80\% efficiency of the nine consecutive arginine PTD used here is comparable to these previously reported efficiencies. The pluripotency factors in the form of recombinant proteins fused with nine consecutive arginines could be used to maintain pluripotency of cESCs in vitro.

In our study, cESCs cultured with the four recombinant pluripotency proteins did not show any differentiation after three generations of culture, neither in morphological shape nor in the expression of marker genes. In contrast, cESCs expressed pluripotency genes until the third generation, when they underwent apoptosis or died, indicating that differentiation was inhibited by the recombinant pluripotency proteins. The requirement for LIF and bFGF reflected the function of the two factors in promoting proliferation of undifferentiated cESCs [23-29]. However, cESCs cultured with a combination of mouse LIF and human bFGF only did not exhibit any positive effect on pluripotency maintenance. This observation was in line with previous studies reporting that cESCs cultured with mouse LIF and human bFGF underwent differentiation after just one passage and indicates that the two factors alone were not as functional in maintaining cESC pluripotency as they were in maintaining mESC and hESC pluripotency [4,23]. A combination of the four recombinant pluripotency proteins with LIF and bFGF showed a powerful ability to support cESC proliferation for at least seven generations. In addition, the time interval between generations was shorter, indicating that cESCs became more vigorous in medium containing both pluripotency proteins and the two factors. These results demonstrated that the four pluripotency proteins complement the function of LIF and bFGF as promoters of proliferation and supporters of cESC pluripotency in vitro. Most genes downstream of LIF and bFGF are the same as those downstream of the pluripotency factors [30-32]. In addition, these downstream genes always undergo epigenetic changes before they are regulated by LIF or bFGF [33-37]. The ability of the LIF or bFGF to promote proliferation could be a result of the maintenance of pluripotency by the added pluripotency proteins, which provided an appropriate epigenetic environment for their signaling pathways to converge and work together. In another respect, the requirement of LIF and bFGF may also reflect an insufficient level of pluripotency proteins, which may have been limited by the total volume that can be held by a culture plate well. There may not have been sufficient pluripotency factors added to the cESC culture medium to fully support both pluripotency and self-renewal, indicating that additional pluripotency proteins might substitute the need for LIF and bFGF. In addition, positive staining for SSEA-I and AKP activity, as well as the expression of pluripotency genes demonstrated the pluripotency of cESCs cultured for six generations. Thus, addition of pluripotency proteins provides a direct and useful way to maintain pluripotency of cESCs in vitro. 
With some improvements, the proliferation of cultured cells could be promoted for a longer time.

Assessment of differentiation ability and telomerase activity provided further convincing evidence of the cultured cESCs' pluripotency and demonstrated the possibility of generating a long-term cESC line using the four recombinant pluripotency proteins. EB structures were formed by cESCs cultured after six generations, and contained various differentiated cells derived from all three embryonic germ layers. Although the EB structure is far from a real embryonic structure in organization and function, this in vitro differentiation test indicated that cESCs of at least six generations have the potential to differentiate into cells of all three embryonic germ layers. Telomerase activity reflects the immortality of cESC and is routinely tested as a determination of pluripotency and self-renewal. TERT is an important catalytic subunit of telomerase and is considered as a marker of telomerase activity $[38,39]$. TERT expression in cultured cESCs showed consistent telomerase activity from zero to six generations, indicating the maintenance of telomerase activity in cultured cESCs.

Pluripotency factors have been reported to function in differentiation inhibition, the maintenance of the open state of chromatin, and protection of the unique short cell cycle of ESCs [40-44]. POUV inhibits ESCs from differentiating into ectoderm cells [45], while Sox-2 forms a temporary complex with POUV when regulating the cell cycle and chromatin state through downstream target genes [46,47]. Levels of Nanog are considered to be a switch leading ESCs to rapidly choose between self-renewal and differentiation [48]. Here, Lin28 exhibited an essential role in cESCs based on the results of pluripotency gene expression tests. In these tests, genes were steadily expressed until the seventh generation, when a decrease of Lin28 expression was detected. This was followed by a decrease in the expression of other pluripotency genes and the termination of cESC proliferation. Lin28 is a blocker of the mRNA Let-7 maturation process. Mature Let-7 binds to mRNAs of target genes involved in cell cycle facilitation, telomerase activity protection, and chromatin reconstruction and silences their expression [49-52]. Lin28 was also reported to stabilize these mRNAs and increase their expression [53-57]. Therefore, Lin 28 could be viewed as a pluripotency factor and an epigenetic factor. It has been widely demonstrated and accepted that when cell cycle progression of cultured ESCs is blocked, chromatin structure condenses, and a series gene expression changes occur allowing ESCs to undergo differentiation or apoptosis [58-61]. Therefore, Lin28, by linking epigenetic status and cell cycle progression of ESCs with pluripotency and self-renewal, should be a central focus for ESC research.

Taken together, chickens offer numerous advantages as transgenic animals for medical, pharmacological and biotechnology research. The generation of a long-term cESC line in vitro has become increasingly necessary. Such a cell line should be stable, highly proliferative, immortal, easily used, and consistent. In this study, a new culture system was developed, being the first to introduce pluripotency factors into the cESC culture method. We have shown the ability of the novel system to maintain the pluripotency of cESCs for at least six generations in vitro and to provide a relatively stable and large number of cells for genetic engineering and other areas of cESC research. The results of this study, together with previous iPSCs reports, demonstrate that ample expression of pluripotency factors are the key to pluripotency and self-renewal of ESCs. With further improvements, a long-term cESC line could be developed in vitro based on this method.

We thank our colleagues of the Laboratory for Molecular and Cellular Genetics for their help in molecular and cell culture work. This work was assisted by the National Key Lab of AgroBiotechnology, and their members should be given great thanks.

1 Evans M, Kaufman M. Establishment in culture of pluripotential cells from mouse embryos. Nature, 1981, 292: 154-156

2 Martin G. Isolation of a pluripotent cell line from early mouse embryos cultured in medium conditioned by teratocarcinoma stem cells. Proc Natl Acad Sci USA, 1981, 78: 7634-7638

3 Petitte J N, Liu G, Yang Z. Avian pluripotent stem cells. Mech Devel, 2004, 121: 1159-1168

4 Li J, PAN Q, Li J, et al. Research of blastocyte-like structure in chicken. Sci China Ser C-Life Sci, 2005, 48: 481-486

5 Speksnijder G, Ivarie R. A modified method of shell windowing for producing somatic or germline chimeras in fertilized chicken eggs. Poult Sci, 2000, 79: 1430-1433

6 Horiuchi H, Tategaki A, Yamashita Y, et al. Chicken leukemia inhibitory factor maintains chicken embryonic stem cells in the undifferentiated state. J Biol Chem, 2004, 279: 24514-24520

7 Nichols J, Evans E P, Smith A G. Establishment of germ-line competent embryonic stem (ES) cells using differentiation inhib-iting activity. Development, 1990, 110: 1341-13484

8 Pain B, Clark M E, Shen $\mathrm{H}$, et al. Long-term in vitro culture and characterisation of avian embryonic stem cells with multiple morphorgenetic potentialities. Development, 1996, 122: 2339-2348

9 Thomson J A, Itskovitz E J, Shapiro S S, et al. Embryonic stem cell lines derived from human blastocysts. Science, 1998, 282: 1145-1147

10 Stadtfeld M, Hochedlinger K, Induced pluripotency: history, mechanisms, and applications. Genes Dev, 2010, 24: 2239-2263

11 Takahashi K, Yamanaka S. Induction of pluripotent stem cells from mouse embryonic and adult fibroblast cultures by defined factors. Cell, 2006, 126: 663-676

12 Takahashi K, Tanabe K, Ohnuki M, et al. Induction of pluripotent stem cells from adult human fibroblasts by defined factors. Cell, 2007, 131: 861-872

13 Kaji K, Norrby K, Paca A, et al. Virus-free induction of pluripotency and subsequent excision of reprogramming factors. Nature, 2009, 458: 771-775.

$14 \mathrm{Yu}$ J, Hu K, Smuga O K, et al. Human induced pluripotent stem cells free of vector and transgene sequences. Science, 2009, 324: 797-801

15 Jia F, Wilson K D, Sun N, et al. A nonviral minicircle vector for deriving human iPS cells. Nat Methods, 2010, 7: 197-199

16 Wender P A, Mitchell D J, Pattabiraman K, et al. The design, synthesis, and evaluation of molecules that enable or enhance cellular uptake: Peptoid molecular transporters. Proc Natl Acad Sci USA, 2000, 97: $13003-13008$ 
17 Ziegler A, Nervi P, Durrenberger M, et al. HIV TAT variants differentially influence the production of glucocerebrosidase in Sf9 cells. Biochemistry, 2005, 44: 138-148

18 Michiue $\mathrm{H}$, Tomizawa $\mathrm{K}$, Wei F Y, et al. The NH2 terminus of influenza virus hemagglutinin-2 subunit peptides enhances the antitumor potency of polyarginine-mediated p53 protein transduction. $\mathbf{J}$ Biol Chem, 2005, 280: 8285-8289

19 Lafevre-Bernt M, Wu S, and Lin X. Recombinant, refolded tetrameric p53 and gonadotropin-releasing hormone-p53 slow proliferation and induce apoptosis in p53-deficient cancer cells. Mol Cancer Ther, 2008, 7: 1420-1429

20 Dohoon K, Chun H K, Jung I M, et al. Generation of human induced pluripotent stem cells by direct delivery of reprogramming proteins. Cell Stem Cell, 2009, 4: 472-476

21 Hongyan Z, Shili W, Jin Y J, et al. Generation of induced pluripotent stem cells using recombinant proteins. Cell Stem Cell, 2009, 8: 1-4

22 Sayed A, Futaki S, Harashima H. Delivery of macromolecules using arginine-rich cell-penetrating peptides: ways to overcome endosomal entrapment. AAPS J, 2009, 11: 13-22

23 Li J, Zhang B, Han H, et al. Metabolic properties of chicken embryonic stem cells. Sci China Life Sci, 2010, 53: 1073-1084

24 Casanova E A, Shakhova O, Patel S S, et al. Pramel7 mediates LIF/STAT3-dependent self-renewal in embryonic stem cells. Stem Cells, 2011, 29: 474-485

25 Shin J E, Park S H, Jang Y K. Epigenetic up-regulation of leukemia inhibitory factor (LIF) gene during the progression to breast cancer. Mol Cells, 2011, 31: 181-189

26 Griffiths D S, Li J, Dawson M A. LIF-independent JAK signalling to chromatin in embryonic stem cells uncovered from an adult stem cell disease. Nat Cell Biol, 2011, 13: 13 - 21

27 Eiselleova L, Matulka K, Kriz V, et al. A complex role for FGF-2 in self-renewal, survival, and adhesion of human embryonic stem cells. Stem Cells, 2009, 27: 1847-1857

28 Greber B, Lehrach H, Adjaye J. Fibroblast growth factor 2 modulates transforming growth factor beta signaling in mouse embryonic fibroblasts and human ESCs (hESCs) to support hESC self-renewal. Stem Cells, 2007, 25: 455-464

29 Greber B, Wu G, Bernemann C. Conserved and divergent roles of FGF signaling in mouse epiblast stem cells and human embryonic stem cells. Cell Stem Cell, 2010, 6: 215-226

30 Kim J, Chu J, Shen X, et al. An extended transcriptional network for pluripotency of embryonic stem cells. Cell, 2008, 132: 1049-1061

31 Chen X, Xu H, Yuan P, et al. Integration of external signaling pathways with the core transcriptional network in embryonic stem cells. Cell, 2008, 133: 1106-1117

32 Boyer L A, Lee T I, Cole M F, et al. Core transcriptional regulatory circuitry in human embryonic stem cells. Cell, 2005, 122: 947-956

33 Reik W. Stability and flexibility of epigenetic gene regulation in mammalian development. Nature, 2007, 447: 425-432

34 Singhal N, Graumann J, Wu G, et al. Chromatin-remodeling components of the BAF complex facilitate reprogramming. Cell, 2010, 141: 943-955

35 Meissner A. Epigenetic modifications in pluripotent and differentiated cells. Nat Biotechnol, 2010, 28: 1079-1088

36 Meshorer E, Misteli T. Chromatin in pluripotent embryonic stem cells and differentiation. Nat Rev Mol Cell Biol, 2006, 7: 540-546

37 Efroni S, Duttagupta R, Cheng J, et al. Global transcription in pluripotent embryonic stem cells. Cell Stem Cell, 2008, 2: 437-447

38 Wang Y, Erdmann N, Giannone R J, et al. An increase in telomere sister chromatid exchange in murine embryonic stem cells possessing critically shortened telomeres. Proc Natl Acad Sci USA, 2005, 102:
10256-10260

39 Blasco M A. The epigenetic regulation of mammalian telomeres. Nat Rev Genet, 2007, 8: 299-309

40 Stadtfeld M, Hochedlinger K. Induced pluripotency: history, mechanisms, and applications. Genes Dev, 2010, 24: 2239-2263

41 Marson A, Levine S S, Cole M F, et al. Connecting microRNA genes to the core transcriptional regulatory circuitry of embryonic stem cells. Cell, 2008, 134: 521-533

42 Meshorer E, Yellajoshula D, George E, et al. Hyperdynamic plasticity of chromatin proteins in pluripotent embryonic stem cells. Dev Cell, 2006, 10: 105-116

43 Spivakov M, Fisher A G. Epigenetic signatures of stem-cell identity. Nat Rev Genet, 2007, 8: 263-271

44 Hanna J H, Saha K, Jaenisch R. Pluripotency and cellular reprogramming: facts, hypotheses, unresolved issues. Cell, 2010, 143: 508-525

45 Niwa H, Toyooka Y, Shimosato D, et al. Interaction between Oct3/4 and $\mathrm{Cdx} 2$ determines trophectoderm differentiation. Cell, 2005, 123: 917-929

46 Kuroda T, Tada M, Kubota H, et al. Octamer and Sox elements are required for transcriptional cis regulation of Nanog gene expression. Mol Cell Biol, 2005, 25: 2475-2485

47 Rodda D J, Chew J L, Lim L H. Transcriptional regulation of nanog by OCT4 and SOX2. J Biol Chem, 2005, 280: 24731-24737

48 Chambers I, Silva J, Colby D, et al. Nanog safeguards pluripotency and mediates germline development. Nature, 2007, 450: 1230-1234

49 Heo I, Joo C, Cho J. Lin28 mediates the terminal uridylation of let-7 precursor MicroRNA. Mol Cell, 2008, 32: 276-284

50 Loughlin F E, Gebert L F, Towbin H, et al. Structural basis of pre-let-7 miRNA recognition by the zinc knuckles of pluripotency factor Lin28. Nat Struct Mol Biol, 2011, 19: 84-89

51 Nam Y, Chen C, Gregory R I. Molecular basis for interaction of let-7 microRNAs with Lin28. Cell, 2011, 174: 1080-1091

52 Melton C, Judson R L, Blelloch R. Opposing microRNA families regulate self-renewal in mouse embryonic stem cells. Nature, 2010, 463: 621-626

53 Reik W. Stability and flexibility of epigenetic gene regulation in mammalian development. Nature, 2007, 447: 425-432

54 Hawkins R D, Hon G C, Yang C, et al. Dynamic chromatin states in human ES cells reveal potential regulatory sequences and genes involved in pluripotency. Cell Res, 2011, 21: 1393-1409

55 Klemke M, Meyer A, Hashemi N M, et al. Loss of let-7 binding sites resulting from truncations of the 3' untranslated region of HMGA2 mRNA in uterine leiomyomas. Cancer Genet Cytogen, 2010, 196: 119-123

56 Hanna J, Saha K, Pando B, et al. Direct cell reprogramming is a stochastic process amenable to acceleration. Nature, 2009, 462: 595-601

57 Li N, Zhong X, Lin X, et al. Lin-28 homologue A (LIN28A) promotes cell cycle progression via the regulation of cyclin-dependent kinase 2 (CDK2), cyclin D1 (CCND1), and cell division cycle 25 homolog A (CDC25A) expression in cancer. J Biol Chem, 2010, 30: $1-24$

58 Meshorer E, Yellajoshula D, George E, er al. Hyperdynamic plasticity of chromatin proteins in pluripotent embryonic stem cells. Dev Cell, 2006, 10: 105-116

59 Meshorer E, Misteli T. Chromatin in pluripotent embryonic stem cells and differentiation. Nat Rev Mol Cell Biol, 2006, 7: 540-546

60 Golob J L, Paige S L, Muskheli V, et al. Chromatin remodeling during mouse and human embryonic stem cell differentiation. Devel Dynamics, 2008, 237: 1389-1398

61 White J, Dalton S. Cell cycle control of embryonic stem cells. Stem Cell Rev, 2005, 1: 131-138

Open Access This article is distributed under the terms of the Creative Commons Attribution License which permits any use, distribution, and reproduction in any medium, provided the original author(s) and source are credited. 